\title{
Orbital Malignant Peripheral Nerve Sheath Tumor Arising from Neurofibroma
}

\author{
Dear Editor,
}

Malignant peripheral nerve sheath tumor (MPNST) is an uncommon soft tissue sarcoma that arises from peripheral nerves. MPNST of the orbit is very rare and only accounts for $0 \%$ to $0.2 \%$ of all orbital tumors. About $70 \%$ of MPNSTs are associated with neurofibromatosis type 1 (NF1) and benign neurofibromas rarely undergo malignant transformation. As far as the authors know there has been only one report of an orbital MPNST arising from a benign neurofibroma. Here, we report a patient with MPNST arising from a benign neurofibroma who showed long survival with surgical excision and radiation.

A 19-year-old male visited our clinic for a left eye proptosis lasting 4 months. On examination, visual acuity in his left eye was $20 / 20$ and exophthalmometric values were $16 \mathrm{~mm}$ on the right eye and $20.5 \mathrm{~mm}$ on the left eye. The exophthalmic left eye showed limited upward gaze. Computed tomography scan revealed an extraconal $22 \times 18 \times$ 42-mm-sized mass between the superior rectus muscle and the superior orbital wall with peripheral wall enhancement and central mottled enhancement (Fig. 1A, 1B). Magnetic resonance imaging with dynamic contrast enhancement showed a well-defined and spindle-shaped mass with low signal intensity on T1-weighted images and high signal intensity on T2-weighted images. After contrast enhancement, mottled enhancement was observed in the central portion of the tumor that gradually increased over time (Fig. 1C, 1D).

Surgical excision was performed through superior orbitotomy. After opening the superior periorbita, the mass was exposed. The mass was carefully dissected and removed. Pathologic examination revealed small round cells with increased cellularity and a fibrillary background. Mitotic count was two per 10 high power fields and the resection margin was associated with a neurofibroma (Fig. 1E, 1F).
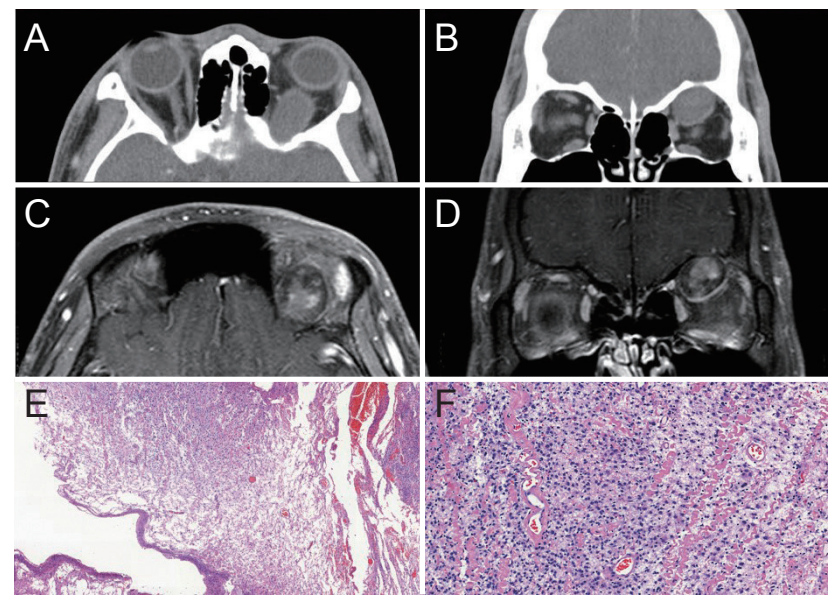

Fig. 1. Radiological and histological appearance of orbital malignant peripheral nerve sheath tumor. (A) Axial and (B) coronal computed tomography scan showing extraconal mass with peripheral wall enhancement and central mottled enhancement. (C) Axial and (D) coronal view of T1-weighted contrast-enhanced with fat suppression magnetic resonance imaging demonstrating a well-defined and ovalshaped mass with low signal intensity, peripheral wall enhancement and central mottled enhancement. (E) Histopathologic finding shows small round cells in fibrillary background transitioning from low to high cellularity (hematoxylin and eosin, $\times 50)(\mathrm{F})$ with active cell division and atypical nuclei (hematoxylin and eosin, $\times 200$ ).

Immunohistochemistry revealed cells positive for vimentin, S-100, neuron specific enolase, suggestive of a neural origin. Immunonegativity of desmin and smooth muscle actin helped rule out the possibility of tumors of muscular origin. Based on these findings, the patient was diagnosed with low grade MPNST in the background of neurofibroma. The patient received adjuvant fractionated stereotactic radiotherapy to the left orbit. He has been disease-free without recurrence until the last follow-up (7 years postoperatively).

MPNSTs are known to originate from cells constituting the nerve sheath including perineural fibroblasts or Schwann cells. NF-1 and irradiation history are known risk factors for MPNST. The prevalence of MPNST in patients with NF-1 is $4 \%$ and $0.001 \%$ in the general population [1]. MPNSTs generally arise de novo but may also arise as a consequence of malignant transformation of a neurofibroma. There is only one reported case of malignant transformation of a neurofibroma in the orbit of a patient without NF-1 and this is a second case [2]. 
On computed tomography, MPNSTs often appear as well-defined, rounded, homogenously enhancing masses and these tumors may be found to erode adjacent bony structures. These findings were also observed in our case. On magnetic resonance imaging, MPNSTs have an isointense homogenous signal intensity on T1-weighted images, and necrotic lesions show high signal intensity on T2-weighted images [3]. In our case, the tumor showed low signal intensity on T1-weighted images and high signal intensity on T2-weighted images.

The definitive diagnosis of MPNST is obtained by biopsy. MPNSTs are highly cellular and composed of spindle-shaped cells which have active cell division and atypical nuclei [4]. Immunohistochemical staining can be helpful for diagnosis when it shows a positive response for neural antigens including vimentin, S-100 protein, neuron-specific enolase and neurofilaments, and a negative response for antibodies associated with differentiation of epithelial cells, neuroendocrine cells, or muscle cells [5].

The main treatment is surgical excision and wide excision of the primary site should be done. If there is intracranial invasion, orbital exenteration with craniotomy is suggested. Radiation therapy is recommended after surgery, especially when complete resection is difficult or when the resection margin is uncertain [2].

We report a case of MPNST arising from a neurofibroma. Our case showed long-term survival after surgical excision and postoperative radiation therapy, and this is thought to be due to complete tumor excision. Although the incidence of MPNST is very rare, it should be included in the differential diagnosis of rapid-growing orbital masses.

Seoyoung Wy

Department of Ophthalmology, Seoul National University Hospital, Seoul, Korea
Namju Kim

Department of Ophthalmology, Seoul National University Bundang Hospital, Seongnam, Korea

E-mail:kimnamju@snubh.org

\section{Conflict of Interest}

No potential conflict of interest relevant to this article was reported.

\section{References}

1. Dutton JJ, Tawfik HA, DeBacker CM, et al. Multiple recurrences in malignant peripheral nerve sheath tumor of the orbit: a case report and a review of the literature. Ophthalmic Plast Reconstr Surg 2001;17:293-9.

2. Cheng SF, Chen YI, Chang CY, et al. Malignant peripheral nerve sheath tumor of the orbit: malignant transformation from neurofibroma without neurofibromatosis. Ophthalmic Plast Reconstr Surg 2008;24:413-5.

3. Stull MA, Moser RP Jr, Kransdorf MJ, et al. Magnetic resonance appearance of peripheral nerve sheath tumors. Skeletal Radiol 1991;20:9-14.

4. Wanebo JE, Malik JM, VandenBerg SR, et al. Malignant peripheral nerve sheath tumors: a clinicopathologic study of 28 cases. Cancer 1993;71:1247-53.

5. Daimaru Y, Hashimoto H, Enjoji M. Malignant peripheral nerve-sheath tumors (malignant schwannomas): an immunohistochemical study of 29 cases. Am J Surg Pathol 1985;9:434-44. 